TRANSACTIONS OF THE

AMERICAN MATHEMATICAL SOCIETY

Volume 355, Number 5, Pages 1921-1931

S 0002-9947(03)03241-0

Article electronically published on January 8, 2003

\title{
HOLOMORPHIC EXTENSIONS FROM OPEN FAMILIES OF CIRCLES
}

\author{
JOSIP GLOBEVNIK \\ Dedicated to Professor Ivan Vidav on the occasion of his eighty-fifth birthday
}

\begin{abstract}
For a circle $\Gamma=\{z \in \mathbb{C}:|z-c|=\rho\}$ write $\Lambda(\Gamma)=\{(z, w):(z-$ $\left.a)(w-\bar{a})=\rho^{2}, 0<|z-a|<\rho\right\}$. A continuous function $f$ on $\Gamma$ extends holomorphically from $\Gamma$ (into the disc bounded by $\Gamma$ ) if and only if the function $F(z, \bar{z})=f(z)$ defined on $\{(z, \bar{z}): z \in \Gamma\}$ has a bounded holomorphic extension into $\Lambda(\Gamma)$. In the paper we consider open connected families of circles $\mathcal{C}$, write $U=\bigcup\{\Gamma: \Gamma \in \mathcal{C}\}$, and assume that a continuous function on $U$ extends holomorphically from each $\Gamma \in \mathcal{C}$. We show that this happens if and only if the function $F(z, \bar{z})=f(z)$ defined on $\{(z, \bar{z}): z \in U\}$ has a bounded holomorphic extension into the domain $\bigcup\{\Lambda(\Gamma): \Gamma \in \mathcal{Q}\}$ for each open family $\mathcal{Q}$ compactly contained in $\mathcal{C}$. This allows us to use known facts from several complex variables. In particular, we use the edge of the wedge theorem to prove a theorem on real analyticity of such functions.
\end{abstract}

\section{INTRODUCTION}

Write $\Delta(a, \rho)=\{\zeta \in \mathbb{C}:|\zeta-a|<\rho\}, \Delta=\Delta(0,1)$. We say that a continuous function on $b \Delta(a, \rho)$ extends holomorphically from $b \Delta(a, \rho)$ if it has a continuous extension to $\bar{\Delta}(a, \rho)$ which is holomorphic on $\Delta(a, \rho)$.

The initial motivation for the work whose results are presented here was the following.

Question [G2]. Let $D=\{\zeta \in \mathbb{C}:|\operatorname{Im} \zeta|<1\}$ and let $f$ be a continuous function on $\bar{D}$. Suppose that $f$ extends holomorphically from every circle $b \Delta(t, 1), t \in \mathbb{R}$. Is $f$ holomorphic on $D$ ?

M. Agranovsky and the author $\mathrm{AG}$ and, independently, L. Ehrenpreis $\mathrm{E}$ have shown that the answer is positive under the additional assumption that $f$ is realanalytic in a neighbourhood of $\bar{D}$. After the present paper was submitted for publication the author received a preprint from A. Tumanov [T] in which he proves that the answer is positive in general.

We begin by an example. Let $\mathcal{C}$ be the family of all circles in $\mathbb{C}$ surrounding the origin. There are continuous functions $f$ on $U=\mathbb{C} \backslash\{0\}$ which extend holomorphically from each $\Gamma \in \mathcal{C}$ and are not holomorphic on $U$. An example is $f(z)=1 / \bar{z}$.

Let $a \in \mathbb{C}$ and $\rho>0$. As in [1], let $\Lambda_{a, \rho}=\left\{(z, w) \in \mathbb{C}^{2}:(z-a)(w-\bar{a})=\rho^{2}, 0<\right.$ $|z-a|<\rho\}$. This is a closed complex submanifold of $\mathbb{C}^{2} \backslash \Sigma$ attached to the real

Received by the editors July 24, 2002.

2000 Mathematics Subject Classification. Primary 30E20.

(C)2003 American Mathematical Society 
two-plane $\Sigma=\{(z, \bar{z}): z \in \mathbb{C}\}$ along the circle $b \Lambda_{a, \rho}=\{(z, \bar{z}): z \in b \Delta(a, \rho)\}$. We call $\Lambda_{a, \rho}$ the variety associated with $b \Delta(a, \rho)$.

It is easy to see that the function $f$ extends holomorphically from $b \Delta(a, \rho)$ if and only if the function $(z, \bar{z}) \mapsto F(z, \bar{z})=f(z)$ defined on $b \Lambda_{a, \rho}$ has a continuous extension to $\Lambda_{a, \rho} \cup b \Lambda_{a, \rho}$ that is holomorphic and bounded on $\Lambda_{a, \rho}$.

We look at open connected familes of circles, that is, at families of the form

$$
\mathcal{C}(\mathcal{P})=\{b \Delta(a, \rho):(a, \rho) \in \mathcal{P}\}
$$

where $\mathcal{P}$ is an open connected subset of $\mathbb{C} \times(0, \infty)$. Denote by $U(\mathcal{P})$ the subset of $\mathbb{C}$ swept by the circles in $\mathcal{C}(\mathcal{P})$ :

$$
U(\mathcal{P})=\bigcup\{\Gamma: \Gamma \in \mathcal{C}(\mathcal{P})\}=\bigcup\{b \Delta(a, \rho):(a, \rho) \in \mathcal{P}\}
$$

and by $\Omega(\mathcal{P})$ the union of the associated varieties $\Lambda_{a, \rho}$ :

$$
\Omega(\mathcal{P})=\bigcup\left\{\Lambda_{a, \rho}:(a, \rho) \in \mathcal{P}\right\} .
$$

We write $\tilde{U}(\mathcal{P})=\{(z, \bar{z}): z \in U(\mathcal{P})\}$, and we write $\mathcal{Q} \subset \subset \mathcal{P}$ if $\mathcal{Q}$ is contained in a compact subset of $\mathcal{P}$.

Suppose that $\mathcal{P}$ is an open connected subset of $\mathbb{C} \times(0, \infty)$, and let $f$ be a continuous function on $\Omega(\mathcal{P})$ which extends holomorphically from each circle in $\mathcal{C}(\mathcal{P})$. In this paper we look at all varieties $\Lambda_{a, \rho},(a, \rho) \in \mathcal{P}$, and simultaneously at holomorphic extensions of $F$ into all these varieties. We show that these extensions match at the intersections of these varieties and give rise to a holomorphic function of two variables in $\Omega(\mathcal{P})$. We show that $\Omega(\mathcal{P})$ is an open connected set attached to $\Sigma$ along $\tilde{U}(\mathcal{P})$, and that the function $f$ extends holomorphically from each circle in $\mathcal{C}(\mathcal{P})$ if and only if for each open set $\mathcal{Q} \subset \subset \mathcal{P}$ the function $F \mid \tilde{U}(Q)$ has a bounded continuous extension to $\Omega(\mathcal{Q}) \cup \tilde{U}(\mathcal{Q})$ that is holomorphic on $\Omega(\mathcal{Q})$. We show that each point of $\tilde{U}(\mathcal{P})$ has a neighbourhood $\tilde{V} \subset \tilde{U}(\mathcal{P})$ such that $\Omega(\mathcal{P})$ contains a wedge with the edge $\tilde{V}$. The function $f$ will be holomorphic on $U(\mathcal{P})$ if and only if $\tilde{F}$ depends only on the first variable. All this allows us to use some known facts from several complex variables when looking at various families of circles $\mathcal{C}(\mathcal{P})$. In particular, we use the edge of the wedge theorem to show that holomorphic extendibility from certain families of circles implies the real analyticity of $f$ (Theorem 6.1).

\section{VARIETIES $\Lambda_{a, \rho}$}

Proposition 2.1. Let $(z, w) \in \mathbb{C}^{2} \backslash \Sigma$. Given $R>0$, there is a unique a such that $(z, w) \in \Lambda_{a, R}:$

$$
a=z+\left[\sqrt{1+4 R^{2} /|z-\bar{w}|^{2}}-1\right](z-\bar{w}) / 2 .
$$

Proof. It is easy to see [1] that $(z, w) \in \Lambda_{a, R}$ if and only if there is a $t>0$ such that $a=z+t(z-\bar{w})$ and $R=\sqrt{t(t+1)}|z-\bar{w}|$. Finding $t$ from $R^{2}=t(t+1)|z-\bar{w}|^{2}$ gives (2.1) and completes the proof.

Proposition 2.2. Let $\left(a_{1}, \rho_{1}\right) \neq\left(a_{2}, \rho_{2}\right)$. Then $\Lambda_{a_{1}, \rho_{1}}$ meets $\Lambda_{a_{2}, \rho_{2}}$ if and only if $a_{1} \neq a_{2}$ and one of the circles $b \Delta\left(a_{1}, \rho_{1}\right), b \Delta\left(a_{2}, \rho_{2}\right)$ surrounds the other. If this is the case, then $\Lambda_{a_{1}, \rho_{1}}$ and $\Lambda_{a_{2}, \rho_{2}}$ meet transversely and the intersection consists of one point. 
Proof. Suppose that $(z, w) \in \Lambda_{a_{1}, \rho_{1}} \cap \Lambda_{a_{2}, \rho_{2}}$. If $a_{1}=a_{2}$, then, by our assumption, $\rho_{1} \neq \rho_{2} ;$ so $\Lambda_{a_{1}, \rho_{1}} \cap \Lambda_{a_{2}, \rho_{2}}=\emptyset$. Consequently $a_{1} \neq a_{2}$. There are $t_{i}>0$ such that $a_{i}=z+t_{i}(z-\bar{w})$ and $\rho_{i}=\sqrt{t_{i}\left(t_{i}+1\right)}|z-\bar{w}|, i=1,2$. Since $a_{1} \neq a_{2}$, it follows that $t_{1} \neq t_{2}$, which implies that one of the circles $b \Delta\left(a_{1}, \rho_{1}\right), b \Delta\left(a_{2}, \rho_{2}\right)$ surrounds the other. By Proposition 2.1, $(z, w)$ is the unique point of $\Lambda_{a_{1}, \rho_{1}} \cap \Lambda_{a_{2}, \rho_{2}}$.

Conversely, assume that one of the circles surrounds the other and that the centers are different. With no loss of generality, assume that the surrounding circle is centered at the origin. So assume that $b \Delta(0, R)$ surrounds $b \Delta(b, \rho), b \neq 0$. Computing the $z$-coordinates of the intersection of the corresponding varieties

$$
w=R_{2} / z \quad(0<|z|<R), \quad w=\bar{b}+\rho^{2} /(z-b) \quad(0<|z-b|<\rho),
$$

we get

$$
(z-b)^{2}+\frac{b \bar{b}+\rho^{2}-R^{2}}{\bar{b}}(z-b)+\rho^{2} \frac{b}{\bar{b}}=0 .
$$

So the roots $z_{1}, z_{2}$ satisfy $\left|z_{1}-b\right|\left|z_{2}-b\right|=\rho^{2}$. Assume for a moment that $\left|z_{1}-b\right|=$ $\rho$; so $z_{1}=b+\rho \zeta$ where $|\zeta|=1$. By $(2.2)$ it follows that $w_{1}=\bar{b}+\rho / \zeta$ and $(b+\rho \zeta)(\bar{b}+\rho / \zeta)=R^{2}$, which is impossible since $|b|+\rho<R$. Thus, one of the roots, say $z_{1}$, satisfies $\left|z_{1}-b\right|<\rho$, and since $|b|+\rho<R$, it follows that $\left|z_{1}\right|<R$. Obviously $z_{1} \neq b, z_{1} \neq 0$ and so $\Lambda_{0, R}$ meets $\Lambda_{a, \rho}$.

Suppose that the varieties $\Lambda_{0, R}$ and $\Lambda_{a, \rho}$ intersect at $(z, w)$ nontransversely. This means that

$$
R^{2} / z=\bar{a}+\rho^{2} /(z-a) \text { and } R^{2} / z^{2}=-\rho^{2} /(z-a)^{2} .
$$

Suppose first that $\rho /(z-a)=+R / z$. The first equality in $(2.3)$ gives $(R-\rho)^{2}=a \bar{a}$. So $R=\rho+|a|$, which is not possible since $b \Delta(R, 0)$ surrounds $b \Delta(a, \rho)$. If $\rho /(z-a)=$ $-R / z$, then the first equality in $(2.3)$ gives $(R+\rho)^{2}=a \bar{a}$; so $R+\rho= \pm|a|$. Neither is possible, since $b \Delta(R, 0)$ surrounds $b \Delta(a, \rho)$. Thus, $\Lambda_{0, R}$ intersects $\Lambda_{a, \rho}$ transversely. This completes the proof.

We will intersect $\Lambda_{a, \rho}$ with two-planes perpendicular to the Lagrangian twoplane $\Sigma$. Note that the two-dimensional subspace perpendicular to $\Sigma$ is $i \Sigma=$ $\{(z,-\bar{z}): z \in \mathbb{C}\}$.

Proposition 2.3. Let $z \in \mathbb{C}, t>0$ and $\varphi \in \mathbb{R}$. Then $(z, \bar{z})+\left(t e^{i \varphi},-t e^{-i \varphi}\right) \in \Lambda_{a, R}$ if and only if $a=z+\sqrt{t^{2}+R^{2}} e^{i \varphi}$.

Proof. By Proposition 2.1, $\left(z+t e^{i \varphi}, \bar{z}-t e^{-i \varphi}\right) \in \Lambda_{a, R}$ if and only if

$$
a=z+t e^{i \varphi}+\left(\sqrt{1+R^{2} / t^{2}}-1\right)\left(2 t e^{i \varphi}\right) / 2=z+\sqrt{t^{2}+R^{2}} e^{i \varphi} .
$$

We will also intersect $\Lambda_{a, R}$ with complex lines $z=$ const.

Proposition 2.4. Let $z \in \mathbb{C}, t>0$ and $\varphi \in \mathbb{R}$. Then $(z, \bar{z})+\left(0, t e^{i \varphi}\right) \in \Lambda_{a, R}$ if and only if

$$
a=z-\frac{2 R^{2}}{\sqrt{4 R^{2}+t^{2}}+t} e^{-i \varphi} .
$$

Proof. By Proposition 2.1, $\left(z, \bar{z}+t e^{i \varphi}\right) \in \Lambda_{a, R}$ if and only if

$$
a=z+\left(\sqrt{1+4 R^{2} / t^{2}}-1\right)\left(-t e^{-i \varphi}\right) / 2=z-\frac{2 R^{2}}{\sqrt{4 R^{2}+t^{2}}+t} e^{-i \varphi} .
$$


If $R>0$ and $a_{1}, a_{2} \in \mathbb{C}, a_{1} \neq a_{2}$, then $\Lambda_{a_{1}, R} \cap \Lambda_{a_{2}, R}=\emptyset$. In fact, $\Lambda_{a_{2}, R}=$ $T\left(\Lambda_{a_{1}, R}\right)$ where $T$ is the translation parallel to $\Sigma$ given by

$$
T(z, w)=\left(z-\left(a_{2}-a_{1}\right), w-\overline{\left(a_{2}-a_{1}\right)} .\right.
$$

\section{Domains $\Omega(\mathcal{P})$}

Let $\mathcal{P}$ be an open subset of $\mathbb{C} \times(0, \infty)$. Proposition 2.1 implies that $\Omega(\mathcal{P})$ is open. In fact, by Proposition 2.1 the set $\bigcup\left\{\Lambda_{b, R}:|b-a|<\delta\right\}$ is an open neighbourhood of $\Lambda_{a, R}$ for each $\delta>0$. Assume, in addition, that $\mathcal{P}$ is connected. We show that $\Omega(\mathcal{P})$ is connected. Since each $\Lambda_{a, \rho}$ is homeomorphic to the punctured disc, it is enough to show that for each $\left(a_{0}, \rho_{0}\right),\left(a_{1}, \rho_{1}\right) \in \mathcal{P}$, there is a path in $\Omega(\mathcal{P})$ joining a point in $\Lambda_{a_{0}, \rho_{0}}$ with a point in $\Lambda_{a_{1}, \rho_{1}}$. Let $\left(a_{0}, \rho_{0}\right),\left(a_{1}, \rho_{1}\right) \in \mathcal{P}$. Since $\mathcal{P}$ is connected, there is a path $(a, \rho):[0,1] \mapsto \mathcal{P}$ such that $(a(0), \rho(0))=\left(a_{0}, \rho_{0}\right),(a(1), \rho(1))=\left(a_{1}, \rho_{1}\right)$. Since $a$ and $\rho$ are bounded on $[0,1]$, it follows that for $w$ large enough, each $\Lambda_{a(t), \rho(t)}$ is a uniformly small perturbation of $\{(z, w): z=a(t)\}, 0 \leq t \leq 1$. Thus we may choose $w_{0}$ so large that the line $\left\{\left(z, w_{0}\right): z \in \mathbb{C}\right\}$ intersects $\Lambda_{a(t), \rho(t)}$ at one point and transversely. Call the intersection point $(z(t), w(t))$. By transversality, $t \mapsto(z(t), w(t))$ is continuous on $[0,1]$ and provides a path joining a point of $\Lambda_{a_{0}, \rho_{0}}$ with a point of $\Lambda_{a_{1}, \rho_{1}}$.

Let $a_{0} \in \mathbb{C}$ and $R>0$. We will need domains of the form $\Omega=\bigcup\left\{\Lambda_{a, R}:\left|a-a_{0}\right|<\right.$ $\delta\}$ for small $\delta>0$. In this case $\Omega=\Omega(Q)$ where $Q=\left\{(a, R):\left|a-a_{0}\right|<\delta\right\}$ is not an open subset of $\mathbb{C} \times(0, \infty)$. We will show that in this case there is a domain $\mathcal{P}$ in $\mathbb{C} \times(0, \infty)$ such that $\Omega=\Omega(\mathcal{P})$. In our case $\bigcup\left\{b \Delta(a, R):\left|a-a_{0}\right|<\delta\right\}$ is the annulus $A=\left\{z \in \mathbb{C}: R-\delta<\left|z-a_{0}\right|<R+\delta\right\}$, and so $\Omega$ is the union of varieties associated with circles of radius $R$ contained in $A$. We shall show that whenever a circle $\Gamma \subset A$ surrounds the hole, the associated variety is contained in $\Omega$. Thus, $\Omega=\Omega(\mathcal{P})$ where $\mathcal{P}=\{(a, \rho) \in \mathbb{C} \times(0, \infty):|a|+\rho<R+\delta,|a|-\rho>-(R-\delta)\}$.

Proposition 3.1. Let $0<\rho<R$. The variety $\Lambda_{0, \rho}$ is contained in the union of varieties $\left\{\Lambda_{a, R}:|a|<R-\rho\right\}$, and this is the smallest family of varieties $\Lambda_{a, R}$ whose union contains $\Lambda_{0, \rho}$.

Remark. In other words, to cover $\Lambda_{a, \rho}$ we have to take the family of varieties associated with all circles of radius $R$ that surround the circle $b \Delta(0, \rho)$.

Proof. Let $0<t<\rho$ and $\alpha \in \mathbb{R}$. Proposition 2.1 implies that the point

$$
\left(t e^{i \alpha}, \rho^{2} e^{-i \alpha} / t\right) \in \Lambda_{0, \rho}
$$

belongs to $\Lambda_{a, R}$ if and only if

$$
\begin{aligned}
a & =e^{i \alpha}\left[t+\left[\sqrt{1+4 R^{2} /\left(\rho^{2} / t-t\right)^{2}}-1\right]\left(t-\rho^{2} / t\right) / 2\right] \\
& =e^{i \alpha} \frac{-2\left(R^{2}-\rho^{2}\right)}{\left(t+\rho^{2} / t\right)+\sqrt{\left(t+\rho^{2} / t\right)^{2}+4\left(R^{2}-\rho^{2}\right)}} .
\end{aligned}
$$

It is easy to see that when $t$ increases from 0 to $\rho$ the expression

$$
\frac{R^{2}-\rho^{2}}{\left(t+\rho^{2} / t\right)+\sqrt{\left(t+\rho^{2} / t\right)^{2}+4\left(R^{2}-\rho^{2}\right)}}
$$

increases from 0 to $R-\rho$, and so $a$ moves along the segment with endpoints $0,-e^{i \alpha}(R-\rho)$ from 0 to $-e^{i \alpha}(R-\rho)$. This completes the proof. 
Proposition 3.2. Let $0<R<\rho$. The variety $\Lambda_{0, \rho}$ is contained in the union of varieties $\left\{\Lambda_{a, R}:|a|<\rho-R\right\}$, and this is the smallest family of varieties $\Lambda_{a, R}$ whose union contains $\Lambda_{0, \rho}$.

Remark. In other words, to cover $\Lambda_{0, \rho}$ we have to take the family of varieties $\Lambda_{a, R}$ associated with all circles of radius $R$ that are surrounded by the circle $b \Delta(0, \rho)$.

Proof. As before, $\left(t e^{i \alpha}, \rho^{2} e^{-i \alpha} / t\right) \in \Lambda_{0, \rho}$ belongs to $\Lambda_{a, R}$ if and only if

$$
a=2 e^{i \alpha} \frac{\rho^{2}-R^{2}}{\left(t+\rho^{2} / t\right)+\sqrt{\left(t+\rho^{2} / t\right)^{2}+4\left(R^{2}-\rho^{2}\right)}} .
$$

Again, as $t$ increases from 0 to $\rho$ the expression

$$
\frac{2 \rho^{2}-R^{2}}{\left(t+\rho^{2} / t\right)+\sqrt{\left(t+\rho^{2} / t\right)^{2}+4\left(R^{2}-\rho^{2}\right)}}
$$

increases from 0 to $\rho-R$, and consequently a moves along the segment with endpoints $0, e^{i \alpha}(\rho-R)$ from 0 to $e^{i \alpha}(\rho-R)$. This completes the proof.

Proposition 3.3. Let $z_{0} \in \mathbb{C}, 0<r<R$, and write $\gamma=(R+r) / 2$. Let $A=\{z \in$ $\left.\mathbb{C}: r<\left|z-z_{0}\right|<R\right\}$. Let $\Omega$ be the union of all $\Lambda_{a, \gamma}$ such that $b \Delta(a, \gamma) \subset A$, and let $\Omega_{1}$ be the union of all $\Lambda_{b, \rho}$ such that $b \Delta(b, \rho) \subset A$ surrounds the hole. Then $\Omega=\Omega_{1}$.

Proof. Obviously $\Omega \subset \Omega_{1}$. To show that $\Omega_{1} \subset \Omega$, let $b \Delta(a, \rho) \subset A$ surround the hole. We have to show that $\Lambda_{a, \rho}$ is contained in the union of $\Lambda_{b, \gamma}$ such that $b \Delta(b, \gamma) \subset A$. This is obvious if $\rho=\gamma$. Suppose that $\rho<\gamma$. By Proposition 3.1, $\Lambda_{b, \rho}$ is contained in the union of all $\Lambda_{a, \gamma}$ such that $b \Delta(a, \gamma)$ surrounds $b \Delta(b, \rho)$. Since $b \Delta(b, \rho)$ surrounds the hole, each such $b \Delta(a, \gamma)$ must surround the hole, and so it must be contained in $A$ since $\gamma=(R+r) / 2$. Suppose now that $\rho>\gamma$. By Proposition 3.2, $\Lambda_{b, \rho}$ is contained in the union of $\Lambda_{a, \gamma}$ such that $b \Delta(a, \gamma)$ is surrounded by $b \Delta(b, \rho)$. In particular, each such $b \Delta(a, \gamma)$ is contained in $\Delta\left(z_{0}, R\right)$, and thus surrounds the hole since $\gamma=(R+r) / 2$. So each such $b \Delta(a, \gamma)$ is contained in $A$. This completes the proof.

Proposition 3.4. Let $z_{0}, r, R, \gamma$ and $\Omega$ be as in Proposition 3.3. Then the boundary of $\Omega$ is the union of $\{(z, \bar{z}): z \in \bar{A}\}$ and all $\Lambda_{a, \gamma}$ such that $b \Delta(a, \gamma)$ is tangent to both $b \Delta\left(z_{0}, r\right)$ and $b \Delta\left(z_{0}, R\right)$.

Proof. This is an easy consequence of Proposition 2.1.

Proposition 3.5. Suppose that $b \Delta\left(a_{1}, \rho_{1}\right) \subset \bar{\Delta}\left(a_{2}, \rho_{2}\right)$ and that $b \Delta\left(a, \rho_{1}\right)$ meets $b \Delta\left(a_{2}, \rho_{2}\right)$ at one point $z_{0}$. There is a $\delta>0$ such that if $\mathcal{P}_{i}=\left\{(a, \rho):\left|a-a_{i}\right|<\right.$ $\left.\delta,\left|\rho-\rho_{i}\right|<\delta\right\}, i=1,2$, then $\Omega\left(\mathcal{P}_{1}\right) \cap \Omega\left(\mathcal{P}_{2}\right)$ is connected. Moreover, there is a neighbourhood $\mathcal{S}$ of $z_{0}$ in $\mathbb{C}$ such that $\Omega\left(\mathcal{P}_{1}\right) \cap \Omega\left(\mathcal{P}_{2}\right)$ contains a wedge with the edge $\tilde{\mathcal{S}}=\{(z, \bar{z}): z \in \mathcal{S}\}$

Remark. A wedge here is a domain of the form $E+[V \cap B]$ where $E$ is an open connected subset of $\Sigma, V$ is an open convex cone in $i \Sigma$ with vertex at the origin and $B$ is an open ball centered at the origin. We call $E$ the edge of the wedge $E+[V \cap B]$. 
Proof. Choose $\delta>0$ so small that no circle in $\mathcal{C}\left(\mathcal{P}_{1}\right)$ surrounds a circle from $\mathcal{C}\left(\mathcal{P}_{2}\right)$ and no center of a circle from $\mathcal{C}\left(\mathcal{P}_{1}\right)$ coincides with a center of a circle from $\mathcal{C}\left(\mathcal{P}_{2}\right)$. Since $\mathcal{P}_{1}, \mathcal{P}_{2}$ are open, it follows that $\Omega\left(\mathcal{P}_{1}\right) \cap \Omega\left(\mathcal{P}_{2}\right)$ is open. Suppose that $\left(z_{1}, w_{1}\right),\left(z_{2}, w_{2}\right) \in \Omega\left(\mathcal{P}_{1}\right) \cap \Omega\left(\mathcal{P}_{2}\right)$. We show that there is a path $(z, w):[0,1] \rightarrow$ $\Omega\left(\mathcal{P}_{1}\right) \cap \Omega\left(\mathcal{P}_{2}\right)$ joining $\left(z_{1}, w_{1}\right)$ and $\left(z_{2}, w_{2}\right)$. There are $\left(b_{1}, r_{1}\right),\left(b_{2}, r_{2}\right) \in \mathcal{P}_{1}$ and $\left(c_{1}, R_{1}\right),\left(c_{2}, r_{2}\right) \in \mathcal{P}_{2}$ such that $\left(z_{i}, w_{i}\right)=\Lambda_{b_{i}, r_{i}} \cap \Lambda_{c_{i}, R_{i}}, i=1,2$. Obviously $b \Delta\left(c_{1}, R_{1}\right)$ surrounds $b \Delta\left(b_{1}, r_{1}\right), b \Delta\left(c_{2}, R_{2}\right)$ surrounds $b \Delta\left(b_{2}, r_{2}\right)$ and $c_{i} \neq b_{i}, i=$ 1,2 . It is easy to choose continuous maps $(b, r):[0,1] \rightarrow \mathcal{P}_{1},(c, R):[0,1] \rightarrow \mathcal{P}_{2}$, $b(0)=b_{1}, b(1)=b_{2}, r(0)=r_{1}, r(1)=r_{2}, c(0)=c_{1}, c(1)=c_{2}, R(0)=R_{1}, R(1)=$ $R_{2}$, such that for each $t, 0 \leq t \leq 1, b \Delta(c(t), R(t))$ surrounds $b \Delta(b(t), r(t))$. By the properties of $\mathcal{P}_{1}$ and $\mathcal{P}_{2}$ we have $b(t) \neq c(t)(0 \leq t \leq 1)$. It follows by Proposition 2.1 that for each such $t, \Lambda_{b(t), r(t)}$ meets $\Lambda_{c(t), R(t)}$ in one point $(z(t), w(t))$ and transversely. The transversality implies that the map $t \mapsto(z(t), w(t))$ is continuous on $[0,1]$. It has all the required properties.

Recall that by Proposition 2.3, $(\zeta, \bar{\zeta})+\left(t e^{i \varphi},-t e^{-i \varphi}\right) \in \Lambda_{a, R}$ for $t>0$ and $\varphi$ real if and only if $a=\zeta+\sqrt{t^{2}+R^{2}} e^{i \varphi}$. There is a $\varphi_{0} \in \mathbb{R}$ such that $z_{0}+\rho_{j} e^{i \varphi_{0}}=$ $a_{j}, j=1,2$. Clearly $\rho_{2}>\rho_{1}$. By the preceding discussion, given $j=1,2$, we have $(\zeta, \bar{\zeta})+\left(t e^{i \varphi},-t e^{-i \varphi}\right) \in \Lambda_{a, \rho_{j}}$ with $t>0$ and $\varphi \in \mathbb{R}$ if and only if $a=$ $\zeta+\sqrt{t^{2}+\rho_{j}^{2}} e^{i \varphi}$.

It is easy to see that there is an $\eta>0$ such that $\zeta+\sqrt{t^{2}+\rho_{1}^{2}} e^{i \varphi} \in a_{1}+\delta \Delta$ and $\zeta+\sqrt{t^{2}+\rho_{2}^{2}} e^{i \varphi} \in a_{2}+\delta \Delta$ provided that

$$
\left|\zeta-z_{0}\right|<\eta, \quad 0<t<\eta, \quad \text { and }\left|\varphi-\varphi_{0}\right|<\eta,
$$

which implies that $(\zeta, \bar{\zeta})+\left(t e^{i \varphi},-t e^{-i \varphi}\right) \in \Omega\left(\mathcal{P}_{1}\right) \cap \Omega\left(\mathcal{P}_{2}\right)$ whenever (3.1) holds. So $\left\{(\zeta, \bar{\zeta})+\left(t e^{i \varphi},-t e^{-i \varphi}\right):\left|\zeta-z_{0}\right|<\eta, \quad 0<t<\eta, \quad\left|\varphi-\varphi_{0}\right|<\eta\right\}$ is a wedge contained in $\Omega\left(\mathcal{P}_{1}\right) \cap \Omega\left(\mathcal{P}_{2}\right)$. This completes the proof.

Remark. Proposition 3.5 implies that if $F_{i}$ are continuous functions on $\Omega\left(\mathcal{P}_{i}\right) \cup \tilde{\mathcal{S}}$ that are holomorphic on $\Omega\left(\mathcal{P}_{i}\right), i=1,2$, and if $F_{1} \equiv F_{2}$ on $\tilde{\mathcal{S}}$, then $F_{1} \equiv F_{2}$ on $\Omega\left(\mathcal{P}_{1}\right) \cap \Omega\left(\mathcal{P}_{2}\right)$.

\section{Holomorphic EXtensions, LOCAL CASE}

Proposition 4.1. A continuous function $f$ on $b \Delta(a, \rho)$ extends holomorphically from $b \Delta(a, \rho)$ if and only if the function $F$ defined on $b \Lambda_{a, \rho}$ by $F(z, \bar{z})=f(z)(z \in$ $b \Delta(a, \rho))$ has a continuous extension to $\Lambda_{a, \rho} \cup b \Lambda_{a, \rho}$ that is bounded and holomorphic on $\Lambda_{a, \rho}$.

Proof. The function $\tilde{f}$ is a holomorphic extension of $f$ to $\Delta(a, \rho)$ if and only if $\tilde{F}\left(z, \bar{a}+\rho^{2} /(z-a)\right)=\tilde{f}(z)(0<|z-a|<\rho)$ is a bounded holomorphic extension of $F$ to $\Lambda_{a, \rho}$.

Remark. The same reasoning implies that whenever $G$ is a continuous function on $\Lambda_{a, \rho} \cup b \Lambda_{a, \rho}$ that is holomorphic and bounded on $\Lambda_{a, \rho}$, then for each $(z, w) \in \Lambda_{a, \rho}$,

$$
|G(z, w)| \leq \max \left\{|G(\xi, \eta)|: \quad(\xi, \eta) \in b \Lambda_{a, \rho}\right\} .
$$

We shall need the following result, proved in [G3].

Lemma 4.2. Let $0<r<R$, let $\gamma=(R+r) / 2$ and let $A=\{z \in \mathbb{C}: r<|z|<R\}$. Let $f$ be a continuous function on $\bar{A}$ which extends holomorphically from every 
circle $b \Delta(a, \gamma)$ tangent to both $b \Delta(0, r)$ and $b \Delta(0, R)$. Then, on $\bar{A}, f$ is a uniform limit of polynomials in $z$ and $1 / \bar{z}$. In particular, $f$ extends holomorphically from every circle contained in $\bar{A}$ that surrounds the hole.

Notice that the assumptions in Lemma 4.2 are that $\bar{A}$ is the union of the circles $e^{i \omega} \Gamma, \omega \in \mathbb{R}$, where $\Gamma$ is a circle that surrounds the origin and is not centered at the origin, and $f$ extends holomorphically from $e^{i \omega} \Gamma$ for each $\omega \in \mathbb{R}$.

Let $0<r<R$ and let $A=\{z \in \mathbb{C}: r<|z|<R\}$. In the rest of this section we shall denote by $\Omega(A)$ the union of all $\Lambda_{a, \rho}$ associated with circles $b \Delta(a, \rho) \subset A$ that surround the origin. By Proposition 3.3, $\Omega(A)$ is equal to the union of all $\Lambda_{a, \gamma}, \gamma=(R+r) / 2$, associated with circles $b \Delta(a, \gamma) \subset A$ that surround the origin.

Here is our local theorem.

Theorem 4.3. Let $0<r<R$ and let $A=\{z \in \mathbb{C}: r<|z|<R\}$. Let $f$ be a continuous function on $\bar{A}$. The following are equivalent:

(i) $f$ extends holomorphically from every circle of radius $(R+r) / 2$ tangent to both $b \Delta(0, r)$ and $b \Delta(0, R)$;

(ii) $f$ extends holomorphically from every circle in $\bar{A}$ surrounding the hole;

(iii) the function $F(z, \bar{z})=f(z)$ defined on $\{(z, \bar{z}): z \in \bar{A}\}$ extends to a bounded continuous function on $\overline{\Omega(A)}$ which is holomorphic on $\Omega(A)$.

Proof. The equivalence of (i) and (ii) follows from Lemma 4.2. Suppose that (iii) holds. Then for each $a, \rho$ such that $b \Delta(a, \rho)$ surrounds the origin the function $F(z, \bar{z})=f(z)$ has a bounded continuous extension from $b \Lambda_{a, \rho}$ to $\Lambda_{a, \rho} \cup b \Lambda_{a, \rho}$ that is holomorphic on $\Lambda_{a, \rho}$, which, by Proposition 4.1, implies that $f$ extends holomorphically from $b \Delta(a, \rho)$. By continuity it follows that this holds for every circle in $\bar{A}$ that surrounds the origin. This proves that (iii) implies (ii). Conversely, suppose that (ii) holds. By Lemma 4.2 it follows that on $\bar{A}$, the function $f$ is a uniform limit of polynomials in $z$ and $1 / \bar{z}$, which implies that on $\{(z, \bar{z}): z \in \bar{A}\}$ the function $F(z, \bar{z})=f(z)$ is a uniform limit of polynomials in $z$ and $1 / w$. Each such polynomial $P(z, 1 / w)$ is a bounded continuous function on $\overline{\Omega(A)}$ which is holomorphic on $\Omega(A)$. For each $\Lambda_{a, \rho} \subset \overline{\Omega(A)}$ such a polynomial is bounded and continuous on $\Lambda_{a, \rho} \cup b \Lambda_{a, \rho}$ and holomorphic on $\Lambda_{a, \rho}$; hence, by the remark after Proposition 4.1, for each $(z, w) \in \Lambda_{a, \rho}$ we have $|P(z, 1 / w)| \leq \max \left\{|P(\xi, 1 / \eta)|: \quad(\xi, \eta) \in b \Lambda_{a, \rho}\right\}$. It follows that $\max \{|P(z, 1 / w)|: \quad(z, w) \in \overline{\Omega(A)}\}=\max \{|P(z, 1 / w)|: \quad(z, w) \in$ $\{(\zeta, \bar{\zeta}): \zeta \in \bar{A}\}\}$, which implies that the uniform convergence of polynomials in $z$ and $1 / w$ on $\{(z, \bar{z}): z \in \bar{A}\}$ implies the uniform convergence to a bounded continuous function on $\overline{\Omega(A)}$. In particular, if on $\bar{A}$ the function $f$ is the uniform limit of a sequence $P_{n}(z, 1 / \bar{z})$, where $P_{n}$ are polynomials, then $P_{n}(z, 1 / w)$ converges uniformly on $\overline{\Omega(A)}$ to a bounded continuous function $\tilde{F}$ that extends $F$ and is holomorphic on $\Omega(A)$. Thus (iii) holds. This completes the proof.

The function algebra on the annulus $\bar{A}$ generated by $z$ and $1 / \bar{z}$ arose naturally in G3. By our Theorem 4.3 this is the algebra of all functions of the form $f(z)=$ $F(z, \bar{z})(z \in \bar{A})$ where $F$ is a bounded continuous function on the closure of the wedge domain $\Omega(A)$ with the edge $\{(z, \bar{z}): z \in A\}$ which is holomorphic on $\Omega(A)$.

Proposition 4.4. Suppose that $f$ satisfies the conditions of Theorem 4.3. Then $f$ is holomorphic on $A$ (and in fact, extends holomorphically into the hole) if and only if the continuous extension $\tilde{F}(z, w)$ of $F(z, \bar{z})=f(z)$ from $\tilde{A}=\{(z, \bar{z}): z \in A\}$ to $\tilde{A} \cup \Omega(A)$ which is holomorphic on $\Omega(A)$ depends only on the variable $z$. 
Proof. Suppose that $f$ is holomorphic on $A$. By (i) the function $f$ extends holomorphically to $\Delta(0, R)$; denote the extension by $\tilde{f}$. The function $(z, w) \mapsto \tilde{f}(z)$ is holomorphic on $\Delta(0, R) \times \mathbb{C}$ and equal to $\tilde{F}(z, w)$ on $\Omega(A)$; so $F$ depends only on the first variable. Conversely, if $\tilde{F}(z, w)$ depends only on the first variable, $\tilde{F}(z, w)=g(z)$, then obviously $g$ is holomorphic on $\Delta(0, R)$ and $g=f$ on $A$. This completes the proof.

In Theorem 4.3, $\Omega(A)$ is an unbounded wedge domain contained in $\mathbb{C}^{2} \backslash \Sigma$ with the edge $\tilde{A}=\{(z, \bar{z}): \quad z \in A\}$ contained in $\Sigma$. The closure of $\Omega(A)$ misses the complex line $\{(z, w): w=0\}$. So we can use the map $\Phi(z, w)=(z, 1 / w)$ to get another version of Theorem 4.3 and thus another description of continuous functions on $\bar{A}$ which extend holomorphically from each circle in $A$ surrounding the origin. The map $\Phi$ maps $\mathbb{C}^{2} \backslash\{(z, w): w=0\}$ biholomorphically onto itself, and the image of $\Sigma \backslash\{(0,0)\}$ under $\Phi$ is the totally real manifold $\mathcal{L}=\{(z, 1 / \bar{z}): z \in \mathbb{C} \backslash\{0\}\}$. If $b \Delta(a, \rho)$ surrounds the origin, then the analytic disc

$$
\begin{aligned}
\Theta_{a, \rho} & =\Phi\left(\Lambda_{a, \rho}\right) \cup\{(a, 0)\} \\
& =\left\{(z, w):(z-a)\left(\frac{1}{w}-\bar{a}\right)=\rho^{2}, 0<|z-a|<\rho\right\} \cup\{(a, 0)\} \\
& =\left\{\left(z, \frac{z-a}{\bar{a} z-\bar{a} a+\rho^{2}}\right):|z-a|<\rho\right\}
\end{aligned}
$$

is contained in $\mathbb{C}^{2} \backslash \mathcal{L}$ and is attached to $\mathcal{L}$ along $b \Theta_{a, \rho}=\left\{(z, 1 / \bar{z}): z \in b \Delta_{a, \rho}\right\}$. Clearly a continuous function $f$ on a circle $b \Delta(a, \rho) \subset A$ that surrounds the origin extends holomorphically from $b \Delta(a, \rho)$ if and only if the function $G(z, 1 / \bar{z})=f(z)$ defined on $b \Theta_{a, \rho}$ has a continuous extension to $\Theta_{a, \rho} \cup b \Theta_{a, \rho}$ which is holomorphic on $\Theta_{a, \rho}$.

A function $f$ is a uniform limit of polynomials in $z$ and $1 / \bar{z}$ on $\bar{A}$ if and only if on $\{(z, 1 / \bar{z}): z \in \bar{A}\}$ the function $G(z, 1 / \bar{z})=f(z)(z \in \bar{A})$ is a uniform limit of polynomials. Let $\Xi(A)$ be the union of all $\Theta_{a, \rho}$ such that $b \Delta(a, \rho) \subset A$ surrounds the origin. $\Xi(A)$ is a domain in $\mathbb{C}^{2} \backslash \mathcal{L}$ attached to $\mathcal{L}$ along $\{(z, 1 / \bar{z}): z \in A\}$. It is foliated by the discs $\Theta_{a, \gamma}$ where $\gamma=(R+r) / 2$ and $b \Delta(a, \gamma) \subset A$.

Here is an equivalent form of (iii) in Theorem 4.3

Theorem 4.5. Each of (i), (ii) and (iii) in Theorem 4.3 is equivalent to

(iv) the function $G(z, 1 / \bar{z})=f(z)$ extends from $\{(z, 1 / \bar{z}): z \in \bar{A}\} \subset \mathcal{L}$ to a continuous function on $\overline{\Xi(A)}$ which is holomorphic on $\Xi(a)$.

Remark. In the next section we will work with large families of circles, and in general there will be no line $(z, w): z \in \mathbb{C}$ missing all $\Lambda_{a, \rho}$. So the above reasoning will not be possible in general.

\section{Holomorphic extensions, general CASe}

In this section we look at large families of circles.

Theorem 5.1. Let $\mathcal{P}$ be an open connected subset of $\mathbb{C} \times(0, \infty)$. Then $\Omega(\mathcal{P})$ is a domain in $\mathbb{C}^{2} \backslash \Sigma$ attached to $\Sigma$ along $\tilde{U}(\mathcal{P})=\{(z, \bar{z}): z \in U(\mathcal{P})\}$. Let $f$ be a continuous function on $U(\mathcal{P})$. Define $F(z, \bar{z})=f(z) \quad(z \in U(\mathcal{P}))$. The following are equivalent:

(i) $f$ extends holomorphically from each $\Gamma \in \mathcal{C}(\mathcal{P})$; 
(ii) for each open set $\mathcal{Q} \subset \subset \mathcal{P}$ the function $F \mid \tilde{U}(\mathcal{Q})$ has a bounded continuous extension to $\Omega(\mathcal{Q}) \cup \tilde{U}(\mathcal{Q})$ which is holomorphic on $\Omega(\mathcal{Q})$.

Proof. It is obvious that (ii) implies (i). Suppose that (i) holds. Then for each $(a, \rho) \in \mathcal{P}$ the function $F$ extends from $b \Lambda_{a, \rho}=\{(z, \bar{z}): z \in b \Delta(a, \rho)\}$ to a bounded continuous function $F_{a, \rho}$ on $\Lambda_{a, \rho} \cup b \Lambda_{a, \rho}$, holomorphic on $\Lambda_{a, \rho}$. This extension is unique and is bounded on $\Lambda_{a, \rho}$ by $\max \left\{|F(z, w)|:(z, w) \in b \Lambda_{a, \rho}\right\}$. Theorem 4.3 implies that for each $\left(a_{0}, \rho_{0}\right) \in \mathcal{P}$ there is an open neighbourhood $\mathcal{W} \subset \mathcal{P}$ of $\left(a_{0}, \rho_{0}\right)$ such that the function $F$ extends from $\tilde{U}(\mathcal{W})=\{(z, \bar{z}): z \in U(\mathcal{W})\}$ to a continuous function on $\tilde{U}(\mathcal{W}) \cup \Omega(\mathcal{W})$ which is holomorphic and bounded on $\Omega(\mathcal{W})$. On each $\Lambda_{a, \rho},(a, \rho) \in \mathcal{W}$, the extension coincides with $F_{a, \rho}$. In particular, if $(a, \rho),\left(a^{\prime}, \rho^{\prime}\right) \in \mathcal{W}$ are such that $\Lambda_{a, \rho}$ and $\Lambda_{a^{\prime}, \rho^{\prime}}$ meet, then $F_{a, \rho}=F_{a^{\prime}, \rho^{\prime}}$ on $\Lambda_{a, \rho} \cap \Lambda_{a^{\prime}, \rho^{\prime}}$. To show that the extensions $F_{a, \rho}$ give rise to a well-defined function $F$ on $\Omega(\mathcal{P})$, we must show that whenever $(a, \rho),\left(a^{\prime}, \rho^{\prime}\right) \in \mathcal{P}$ are such that $\Lambda_{a, \rho}$ and $\Lambda_{a^{\prime}, \rho^{\prime}}$ meet, then $F_{a, \rho}=F_{a^{\prime}, \rho^{\prime}}$ on $\Lambda_{a, \rho} \cap \Lambda_{a^{\prime}, \rho^{\prime}}$. This is obvious if $\Lambda_{a, \rho}=\Lambda_{a^{\prime}, \rho^{\prime}}$. If $\Lambda_{a, \rho} \neq \Lambda_{a^{\prime}, \rho^{\prime}}$ and $\Lambda_{a, \rho}$ and $\Lambda_{a^{\prime}, \rho^{\prime}}$ meet, then by Proposition 2.2 they meet at one point and transversely; and this happens if and only if the circles $b \Delta(a, \rho), b \Delta\left(a^{\prime}, \rho^{\prime}\right)$ have different centers and one surrounds the other. With no loss of generality assume that $b \Delta(a, \rho)$ surrounds $b \Delta\left(a^{\prime}, \rho^{\prime}\right)$. Since $\mathcal{P}$ is open and connected, there are an $\left(a_{0}, \rho_{0}\right) \in \mathcal{P}$ and a real analytic map $t \mapsto(A(t), R(t))$ from $[0,1]$ to $\mathcal{P}$ such that

(a) $b \Delta\left(a_{0}, \rho_{0}\right) \subset \bar{\Delta}(a, \rho)$ and $b \Delta\left(a_{0}, \rho_{0}\right)$ meets $b \Delta(a, \rho)$ at one point $z_{0}$,

(b) $A(0)=a_{0}, R(0)=\rho_{0}, A(1)=a^{\prime}, R(1)=\rho^{\prime}$,

(c) for each $t, 0<t \leq 1, b \Delta(a, \rho)$ surrounds $b \Delta(A(t), R(t))$, and

(d) $A(t) \neq a(0 \leq t \leq 1)$.

Define $\{(z(t), w(t))\}=\Lambda_{A(t), R(t)} \cap \Lambda_{a, \rho} \quad(0<t \leq 1), \quad(z(0), w(0))=\left(z_{0}, \overline{z_{0}}\right)$. Since $\Lambda_{A(t), R(t)}$ are complex manifolds depending in a real analytic way on $t$, transversality implies that $t \mapsto(z(t), w(t))$ is real analytic on $(0,1]$, and it is easy to see that it is continuous at $t=0$.

For each $(b, r) \in \mathcal{P}$ there is an open neighbourhood $\mathcal{V}$ of $(b, r)$ in $\mathcal{P}$ such that the function $F$ defined on $\Omega(\mathcal{V})$ by $F \mid \Lambda_{a, \rho}=F_{a, \rho}((a, \rho) \in \mathcal{V})$ is well defined and holomorphic on $\Omega(\mathcal{V})$. It follows that the functions $t \mapsto F_{A(t), R(t)}(z(t), w(t))$ and $t \mapsto F_{a, \rho}(z(t), w(t))$ are real analytic on $(0,1]$. Proposition 3.5 implies that there is an $\varepsilon>0$ such that

$$
F_{A(t), R(t)}(z(t), w(t))=F_{a, \rho}(z(t), w(t)) \quad(0<t<\varepsilon) .
$$

So the real analyticity implies that (5.1) holds for all $t, 0 \leq t \leq 1$. In particular, for $t=1$ we obtain $F_{a^{\prime}, \rho^{\prime}}\left|\Lambda_{a^{\prime}, \rho^{\prime}} \cap \Lambda_{a, \rho}=F_{a, \rho}\right| \Lambda_{a^{\prime}, \rho^{\prime}} \cap \Lambda_{a, \rho}$, which we wanted to show. This completes the proof.

\section{HolOmorPhic EXTENSIONS FROM CIRCLES AND REAL ANALYTICITY}

Let $0<r<R$, let $A=\{z \in \mathbb{C}: r<|z|<R\}$, and let $f$ be a continuous function on $A$ which extends holomorphically from each circle $\Gamma \subset A$ surrounding the origin. Then $f$ is not necessarily smooth on $A$. We show this by an example. For $z=r e^{i \theta} \neq 0$ define

$$
f(z)=\sqrt{1+e^{2 i \theta}} .
$$

After choosing the branch of the square root, the function $f$ is well defined and continuous on $\mathbb{C} \backslash\{0\}$. It is constant on each ray emanating from the origin, and 
is not smooth along $\{ \pm i t: t>0\}$. Since $e^{i \theta} \mapsto \sqrt{1+e^{i \theta}}$ is the boundary function of the function $\zeta \mapsto \sqrt{1+\zeta}$ that belongs to the disc algebra, it follows that on $b \Delta, \sqrt{1+\zeta}$ is a uniform limit of polynomials in $\zeta$. In particular, on $\mathbb{C} \backslash\{0\}$, $f(z)=\sqrt{1+z / \bar{z}}$ is a uniform limit of polynomials in $z / \bar{z}$, and consequently $f$ extends holomorphically from every circle surrounding the origin.

The example also shows that such a function can be real analytic on a part of $A$ without being real analytic everywhere on $A$.

Theorem 6.1. Let $\Gamma_{1}, \Gamma_{2}, \Gamma_{3}$ be three circles passing through the origin such that the triangle with vertices at their centers contains the origin. Let $f$ be a continuous function on a neighbourhood of $\Gamma_{1} \cup \Gamma_{2} \cup \Gamma_{3}$ which extends holomorphically from each circle sufficiently close to any of the circles $\Gamma_{1}, \Gamma_{2}, \Gamma_{3}$. Then $f$ is real analytic in a neighbourhood of the origin.

Remark. By Lemma 4.2 it is enough to assume the holomorphic extendibility from circles $\Gamma$ such that those near $\Gamma_{i}$ have the same radius as $\Gamma_{i}, 1 \leq i \leq 3$.

Corollary 6.2. Let $\Gamma_{1}, \Gamma_{2}$ be two circles passing through the origin such that the open discs they surround are disjoint. Suppose that $f$ is a continuous function in a neighbourhood of $\Gamma_{1} \cup \Gamma_{2}$ which extends holomorphically from each circle sufficiently close to either $\Gamma_{1}$ or $\Gamma_{2}$. Then $f$ is real analytic in a neighbourhood of the origin.

Proof of Theorem 6.1. Moving the circles slightly if necessary, we may assume with no loss of generality that the triangle with vertices at the centers contains the origin in its interior. Let $\delta>0$, let $\Gamma_{i}=b \Delta\left(a_{i}, \rho_{i}\right), \mathcal{P}_{i}=\left\{(a, \rho):\left|a-a_{i}\right|<\delta,\left|\rho-\rho_{i}\right|<\right.$ $\delta\}, 1 \leq i \leq 3$, and suppose that $f$ is a continuous function on $U\left(\mathcal{P}_{1}\right) \cup U\left(\mathcal{P}_{2}\right) \cup U\left(\mathcal{P}_{3}\right)$ which extends holomorphically from each $b \Delta(a, \rho), \quad(a, \rho) \in \mathcal{P}_{1} \cup \mathcal{P}_{2} \cup \mathcal{P}_{3}$. For each $i, 1 \leq i \leq 3$, apply Theorem 5.1 to show that the function $F$, defined by $F(z, \bar{z})=\bar{f}(z)$, has a continuouus extension from $\tilde{U}\left(\mathcal{P}_{i}\right)=\left\{(z, \bar{z}): z \in U\left(\mathcal{P}_{i}\right)\right\}$ to $\tilde{U}\left(\mathcal{P}_{i}\right) \cup \Omega\left(\mathcal{P}_{i}\right)$ that is holomorphic on $\Omega\left(\mathcal{P}_{i}\right)$.

Fix $j, 1 \leq j \leq 3$. By Proposition 2.3 there is a $\gamma_{j}>0$ such that if $D_{j}=$ $\left\{(\zeta, \bar{\zeta}):|\zeta|<\gamma_{j}\right\}$ and $V_{j}=\left\{t\left(e^{i \varphi},-e^{i \varphi}\right): 0<t<\gamma_{j},\left|\varphi-\arg a_{j}\right|<\gamma_{j}\right\}$, then the wedge domain $D_{j}+V_{j}$ is contained in $\Omega\left(\mathcal{P}_{j}\right)$. So there is a $\delta>0$ such that if $D=\{(\bar{\zeta}):|\zeta|<\delta\}$ and $W_{j}=\left\{t\left(e^{i \varphi},-e^{i \varphi}\right): 0<t<\delta,\left|\varphi-\arg a_{j}\right|<\gamma\right\}$, then the $W_{i}$ are pairwise disjoint and $F$ has a continuous extension to

$$
D \cup\left(D+W_{1}\right) \cup\left(D+W_{2}\right) \cup\left(D+W_{3}\right),
$$

which is holomorphic on $\left(D+W_{1}\right) \cup\left(D+W_{2}\right) \cup\left(D+W_{3}\right)$.

Recall that $D$ is a subset of $\Sigma$, a Lagrangian subspace of $\mathbb{C}^{2}$, and $W_{j} \subset i \Sigma$ are, by our assumptions about the centers $a_{j}$, such that the convex hull of $W_{1} \cup W_{2} \cup W_{3}$ is all of $i \Sigma$. Hence by Epstein's generalization of the edge of the wedge theorem $[\overline{\mathrm{R}}, F(z, \bar{z})=f(z)$ extends holomorphically into a neighbourhood of the origin in $\mathbb{C}^{2}$. This completes the proof.

Remark. Note that the conditions about position of the centers cannot be dropped. Indeed, some condition of this sort is necessary, as can be seen from example (6.1). Let $f$ be as in (6.1), let $t>0$ be very small and let $c>0$ be very large. Let $\Gamma_{+}$and $\Gamma_{-}$be the circles passing through the point it with centers at $c,-c$, respectively. Then $f$ extends holomorphically from each circle sufficiently close to either $\Gamma_{+}$or $\Gamma_{-}$, yet $f$ is not smooth in a neighbourhood of the point $i t$. 
This work was supported in part by a grant from the Ministry of Education, Science and Sport of the Republic of Slovenia.

\section{REFERENCES}

[AG] M. Agranovsky and J. Globevnik: Analyticity on circles for rational and real-analytic functions of two real variables. J. d'Analyse Math., to appear.

[E] L. Ehrenpreis: Three problems at Mount Holyoke. Radon Transforms and Tomography. Contemp. Math. 278 (2001) 123-130. MR 2002d:44002

[G1] J. Globevnik: Analyticity on rotation invariant families of curves. Trans. Amer. Math. Soc. 280 (1983) 247-254. MR 85f:30060

[G2] J. Globevnik: Analyticity on families of curves. Talk at Bar Ilan University, November 1987

[G3] J. Globevnik: Testing analyticity on rotation invariant families of curves. Trans. Amer. Math. Soc. 306 (1988) 401-410. MR 89g:30078

[G4] J. Globevnik: Holomorphic extensions and rotation invariance. Complex Variables Theory Appl. 24 (1993) 49-51. MR 95g:30002

[R] W. Rudin: Lectures on the edge of the wedge theorem. Conf. Ser. in Math. No. 6. American Mathematical Society, Providence, RI, 1971. MR 46:9389

[T] A. Tumanov: A Morera type theorem in the strip. Preprint.

Institute of Mathematics, Physics and Mechanics, University of Ljubljana, LjubLJANA, Slovenia

E-mail address: josip.globevnik@fmf.uni-lj.si 